\title{
The X-ray Outbursts of Be/X-ray Transients
}

\author{
Ignacio Negueruela
}

SAX SDC, c/o Nuova Telespazio, via Corcolle 19, I00131 Rome, Italy

Atsuo T. Okazaki

Faculty of Engineering, Hokkai-Gakuen University,Toyohira-ku, Sapporo 062-8605, Japan

\begin{abstract}
We present a new scenario for the behaviour of $\mathrm{Be} / \mathrm{X}$-ray binaries based on long-term multiwavelength monitoring and the decretion disc model. The circumstellar discs of the primaries are truncated because of the tidal and resonant effect of the neutron star. The geometry of the systems and the value of viscosity determine the presence or absence of Type I X-ray outbursts. The interaction of a strongly disturbed disc with the neutron star originates Type II X-ray and optical outbursts.
\end{abstract}

\section{Introduction}

$\mathrm{Be} / \mathrm{X}$-ray binaries are composed of a neutron star orbiting a Be star and accreting from its circumstellar disc. The high-energy radiation is believed to arise due to accretion of material associated with the Be star by the compact object (see Negueruela 1998). Be/X-ray transients undergo periods in which the X-ray luminosity suddenly increases by a factor $\gtrsim 10$, which are termed outbursts. There are two different kinds of outbursts:

- Short (a few days) X-ray outbursts $\left(L_{\mathrm{x}} \approx 10^{36}-10^{37} \mathrm{erg} \mathrm{s}^{-1}\right)$ occurring in series separated by the orbital period (Type I or normal), generally (but not always) close to the time of periastron passage of the neutron star.

- Giant (or Type II) X-ray outbursts $\left(L_{\mathrm{x}} \gtrsim 10^{37} \mathrm{erg} \mathrm{s}^{-1}\right.$ ), which do not correlate clearly with orbital parameters and last several weeks.

Those systems with fast-spinning neutron stars do not show pulsed X-ray emission during quiescence because of the centrifugal inhibition of accretion (Stella et al. 1986). Systems with more slowly rotating pulsars show X-ray emission at a level $L_{\mathbf{x}} \lesssim 10^{35} \mathrm{erg} \mathrm{s}^{-1}$ when in quiescence.

\section{Radial outflows vs. quasi-Keplerian discs}

Waters et al. (1989) tried to model the X-ray luminosities of Be/X-ray transients during outbursts making use of a simple wind accretion model in which the neutron star accretes from a relatively fast radial outflow. The density in the 
disc of the Be primary was assumed to follow a power law, as in Waters (1986) model for Be stars. In this scenario, the most relevant parameter is the relative velocity between the outflow and the neutron star, since the X-ray luminosity can then be expressed as

$$
L_{\mathrm{x}}=4 \pi G^{3} M_{\mathrm{x}}^{3} R_{\mathrm{x}}^{-1} v_{\mathrm{rel}}^{-4} F_{m} \propto \rho v_{\mathrm{rel}}^{-3}
$$

where $M_{\mathrm{x}}$ and $R_{\mathrm{x}}$ are the mass and radius of the neutron star and $F_{m}=\rho v_{\text {rel }}$ is the mass flow. In order to explain the wide range of observed X-ray luminosities, large changes in the outflow velocity have to be invoked. For example, the relative velocity must have been $v_{\text {rel }} \approx 300 \mathrm{~km} \mathrm{~s}^{-1}$ during a Type I outburst of $\mathrm{V} 0332+53$ in 1983 . However, it was $\ll 100 \mathrm{~km} \mathrm{~s}^{-1}$ during a Type II outburst in 1973.

There is a large number of implicit assumptions in this formulation, some of which are difficult to justify, but two obvious problems stand up. The first one is the low-luminosity X-ray emission displayed by many $\mathrm{Be} / \mathrm{X}$-ray transients when they are not in outburst (for example, several detections of A $0535+26$ at luminosities of $\approx 2 \times 10^{35} \mathrm{erg} \mathrm{s}^{-1}$ ). The model does not offer any explanation as to why there could be a change from quiescence to outburst, unless very large and sudden changes in the density and velocity of the flow are assumed, while optical and infrared observations do not show any sign of the large variations that would be associated with a change of several orders of magnitude in the density of material. But the major objection to the model is simply the fact that there is no observational evidence whatsoever supporting the existence of such fast outflows. All observations of Be discs imply bulk outflow velocities smaller than a few $\mathrm{km} \mathrm{s}^{-1}$. The evidence for rotationally dominated quasi-Keplerian discs around Be stars is overwhelming (see Hanuschik et al. 1995), specially due to the success of the one-armed global oscillation model to explain V/R variability in the emission lines of Be stars (see Okazaki 1991, 1997; Hummel \& Hanuschik 1997).

The discovery by Reig et al. (1997) of a correlation between the maximum equivalent width reached by the $\mathrm{H} \alpha$ emission line in $\mathrm{Be} / \mathrm{X}$-ray binaries and their orbital period strongly suggested that the neutron star had some kind of effect on the disc of the Be primary. This, together with the increasing evidence for quasi-Keplerian discs around Be stars, has prompted us to investigate whether the properties of $\mathrm{Be} / \mathrm{X}$-ray binaries can be better explained if we assume that the disc surrounding the Be primary is a viscous decretion disc. Due to space limitations, we will not try to argue the case here, but refer the interested reader to future publications (Negueruela \& Okazaki 1999; Negueruela \& Okazaki, in preparation) and concentrate on the derived model. We just note that the choice of the viscous decretion disc model has been prompted by its success at explaining observational characteristics of Be stars (Lee et al. 1991; Porter 1999), but it is not a necessary condition for the majority of the conclusions below. As a matter of fact, the tidal and resonant interaction of the neutron star can effectively truncate the Be star disc for any model in which the outflow velocity in the disc is subsonic. 


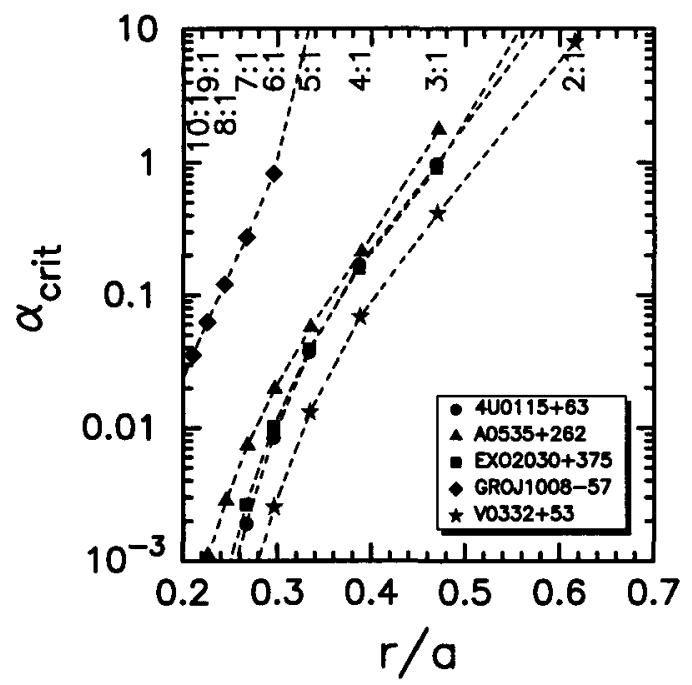

Figure 1. Values of $\alpha_{\text {crit }}$ for several Be/X-ray binaries. The lower axis shows the distance from the central star (normalised to the mean orbital separation $a$ ) at which truncation will occur for a given viscosity. The position of the $n: 1$ commensurabilities is also indicated.

\section{Disc truncation}

A viscous decretion disc is held by the outwards diffusion of angular momentum due to viscous interaction. This viscous torque communicates angular momentum to the outflowing material, allowing it to follow quasi-Keplerian orbits. A neutron star orbiting the Be star exerts a negative torque on the material which takes away angular momentum. The competing effects of viscosity and resonant torques determine the radial distance at which the disc will be truncated. The effect of the neutron star is most strongly felt at the $n: 1$ commensurabilities between the orbits of the neutron star and material at that distance, and truncation is likely to take place there. For every resonance $n: 1$, there is a critical value of viscosity, $\alpha_{\text {crit }}$, such that if $\alpha<\alpha_{\text {crit }}$, the disc is truncated at that resonance. The value of $\alpha_{\text {crit }}$ depends on the orbital parameters of the system under consideration. Fig. 1 shows the values of $\alpha_{\text {crit }}$ that will cause truncation at a given resonance radius for a number of $\mathrm{Be} / \mathrm{X}$-ray binaries. The orbital parameters have been taken from literature (see Negueruela 1998 for references). Those for GRO J1008-57 are based on the best fit to the BATSE data (M. Scott, priv. comm.). The spectral type of GRO J1008-57 and EXO 2030+375 have been assumed to be B0V $\left(M=18 \mathrm{M}_{\odot}\right)$. For reasonable values for the viscosity $(\alpha \sim 0.1)$, we find that the discs in all systems will be truncated at radii very close to the size of the effective Roche radius at periastron.

For the systems with close orbits the first Lagrangian point $L_{1}$ is always further away than the $3: 1$ resonance, indicating that the accretion of large amounts of material will not be possible during quiescence and Type I outbursts will not take place. In A $0535+26$, on the other hand, $r\left(L_{1}\right)<r(3: 1)$ close 
to periastron, while $r\left(L_{1}\right)>r(4: 1)$ always. Similarly, for GRO J1008-57 $r\left(L_{1}\right)<r(6: 1)$ close to periastron, while $r\left(L_{1}\right)>r(7: 1)$ always.

The scenario suggested by these results is as follows. For close systems, the truncation of the disc prevents the accretion of significant amounts of material by the neutron star. Even though the truncation is not expected to be $100 \%$ effective, centrifugal inhibition of accretion for very low accretion rates (Stella et al. 1986) leads to the absence of quiescence X-ray luminosity. However, these systems can still present series of Type I outbursts if the discs surrounding the central Be stars are very disturbed. If some sort of perturbation, such as a global density wave (Okazaki 1997) or radiation-induced warping (Porter 1998), produces an eccentric disc, then material can be accreted through $L_{1}$ close to periastron. This situation will lead to short and irregular series of Xray outbursts, very likely showing decreasing intensity as in the 1996 series of outbursts of $4 U 0115+63$ (Negueruela et al. 1998). Systems with wider orbits, such as A $0535+26$ and GRO J1008-57 can show series of Type I outbursts if the viscosity is high enough to allow the disc to extend beyond $r\left(L_{1}\right)$. Small changes in the viscosity can result in the system switching on or off for relatively long periods. The outbursts in one series will be of approximately the same strength, unless some other effects are involved (it is not clear, for example, how a mediating accretion disc could affect the mass transfer).

The scenario seems to reproduce well the observed properties of most of the systems. Both $4 \mathrm{U} 0115+63$ and V $0332+53$ show extended periods of quiescence during which no X-ray emission at all is detected. V $0332+53$ seems to have kept a very small disc for the last $\sim 7$ years (Negueruela et al. 1999). The outer rim of the $\mathrm{H} \alpha$ emitting region, as determined from the peak separation, is at a distance similar to our calculation for the $4: 1$ resonance. $4 \mathrm{U} 0115+63$ has only once displayed a series of Type I outbursts in $\sim 30$ years of observations and this was when the disc of the Be star was very disturbed (Negueruela et al. 1998). V $0332+53$ has only displayed a series of three Type I outbursts since its discovery. Another source with comparable behaviour is 2 S 1417-62, which has similar orbital parameters. Again, this source has only displayed one series of Type I outbursts and this was following a Type II outburst.

On the other hand, A $0535+26$ shows longer series of Type I outbursts as well as long periods of quiescence (during which it displays low-luminosity X-ray emission). The change between these two states can be explained as a consequence of small variations in the physical conditions in the disc which result in the outer edge moving between the $3: 1$ and $4: 1$ resonances. The outbursts in one series will be of approximately the same strength, unless some other effects are involved. GRO J1008-57 should behave in a similar way.

It must be pointed out that the behaviour of EXO $2030+375$ is very different from that predicted, since it displays very long series of Type I outbursts, similar to those predicted for systems with larger eccentricities. In principle, it could be argued that this is an indication of very high viscosity $(\alpha \sim 1)$, but we note that there is no observational knowledge about the stellar parameters of the central star, which is too heavily obscured to allow even an approximate spectral classification. The actual values for the mass and radius of the Be star could be very different from those used here. 


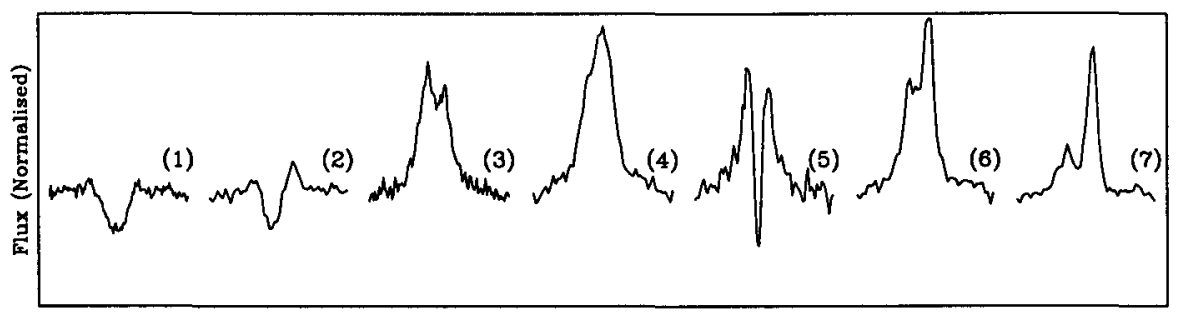

Figure 2. A hypothetical cycle for the $\mathrm{H} \alpha$ line in V635 Cas. Profiles 1-4 are from 1997-1998 (present cycle). Profiles 5-7 are from 19951996 (end of previous cycle). The cycle starts with the absence of the circumstellar disc (profile 1). When the disc appears (2), it quickly grows into a typical double-peaked profile (3). Gradually, the peaks converge until a single-peak profile is seen (4), indicating the warping of the disc. A number of fast transitions between single-peaked and shell profiles (5), indicates precession of the warped disc. Finally, the disc is very perturbed and the asymmetry grows as the strength of the emission line decreases $(6 \& 7)$, leading to a new disc-less phase.

\section{Disc dynamics and Type II outbursts}

Due to truncation, the discs in $\mathrm{Be} / \mathrm{X}$-ray binaries cannot reach a steady state. Material, having lost the angular momentum needed to outflow, will fall back and form a dense torus close to the truncation resonance. This situation will lead to the dynamical instability of the disc which will produce large-scale perturbations, such as warping or global density waves. Such perturbations will either lead to the dispersion of the disc or settle down after some time. Therefore we expect the discs to undergo cycles of reformation and dissipation and/or major perturbations and resettling.

In the case of $\mathrm{V} 635$ Cas, the optical counterpart to $4 \mathrm{U} 0115+63$, spectroscopic monitoring has shown that the Be star undergoes cycles of disc reformation, warping and dissipation with time-scales of 3-5 years (see Figure 2). These cycles are reflected in the X-ray behaviour of the source, which has shown quasi-periodicities of $\sim 3$ years, associated with the main cycle, and $\sim 6-8$ months, when the perturbed disc is precessing. X-ray activity occurs only after the disc is warped, showing that it is the perturbation in the disc which leads to the outburst, and not otherwise (see Negueruela et al. 1998). Estimates of the accretion rates during Type II outbursts show that a significant fraction of the disc material has to be accreted by the neutron star. Since the disc is truncated, accretion of large amounts of matter will only be possible if the disc has become sufficiently asymmetric and dense to overflow the truncation radius.

The large perturbation in the disc of V725 Tau, the optical counterpart to A $0535+26$, which occurred at the time of its last Type II outbursts (Negueruela et al. 1998) has led to the gradual dissipation of the circumstellar envelope (sie Haigh et al. in this proceedings). Such behaviour is reminiscent of that observed 
in V635 Cas and is probably indicating some similarly quasi-cyclical activity on a longer time-scale.

\section{The global view}

The model outlined in this paper provides a global picture, if still sketchy, in which the whole phenomenology of $\mathrm{Be} / \mathrm{X}$-ray binaries is seen as deriving from a small set of simple physical facts. The truncation of the discs surrounding the Be stars by the neutron star companions provides an explanation for the long periods of quiescence, while the dependence of the truncation radius on the physical properties of the disc gives a natural way of understanding the onset of the series of Type I outbursts and their eventual disappearance. Our modelling of different systems shows that in all cases the parameters involved are such that values of the viscosity in the range expected from theoretical considerations and modelling $(0.01<\alpha<1)$ result in truncation at distances comparable to the size of the effective Roche lobe of the Be star. Moreover, in all cases, small changes of the viscosity result in variations in the distance at which truncation takes place. As a consequence of disc truncation, the circumstellar discs cannot be steady, which will lead to the development of the perturbations that have been observed. When the perturbations give rise to large asymmetries in the density distribution, the truncation mechanism will be much less effective and large amounts of material will be able to make their way to the neutron star, producing the giant Type II outbursts.

Acknowledgments. IN is supported by an ESA external fellowship.

\section{References}

Hanuschik, R.W., Hummel, W., Dietle, O., Sutorius, E., 1995, A\&A 300, 163

Hummel, W., Hanuschik, R.W. 1997, A\&A 320, 852

Lee, U., Saio, H, Osaki, Y. 1991, MNRAS 250, 432

Negueruela, I. 1998, A\&A 338, 505

Negueruela, I., Okazaki, A.T. 1999, A\&A, accepted

Negueruela, I., Reig, P., Coe, M.J., Fabregat, J. 1998, A\&A 336, 251

Negueruela, I., Roche, P., Fabregat, J., Coe, M.J. 1999, MNRAS 307, 695

Okazaki, A.T. 1991, PASJ 43, 75

Okazaki, A.T. 1997, A\&A 318, 548

Porter, J.M. 1998, A\&A 336, 966

Porter, J.M. 1999, A\&A 348, 512

Reig, P., Fabregat, J., Coe M.J. 1997, A\&A 322, 193

Stella, L., White, N.E., Rosner, R. 1986, ApJ 308, 669

Waters, L.B.F.M. 1986, A\&A 162, 121

Waters, L.B.F.M., de Martino, D., Habets, G.M.H.J., Taylor, A.R. 1989, A\&A 223,207 